PROCEEDINGS OF THE

AMERICAN MATHEMATICAL SOCIETY

Volume 139, Number 9, September 2011, Pages 3057-3066

S 0002-9939(2011)10734-2

Article electronically published on January 19, 2011

\title{
HIGHER COHEN-MACAULAY PROPERTY OF SQUAREFREE MODULES AND SIMPLICIAL POSETS
}

\author{
KOHJI YANAGAWA \\ (Communicated by Bernd Ulrich)
}

\begin{abstract}
Recently, G. Fløystad studied higher Cohen-Macaulay property of certain finite regular cell complexes. In this paper, we partially extend his results to squarefree modules, toric face rings, and simplicial posets. For example, we show that if (the corresponding cell complex of) a simplicial poset is $l$-Cohen-Macaulay, then its codimension one skeleton is $(l+1)$-CohenMacaulay.
\end{abstract}

\section{INTRODUCTION}

Let $\mathcal{X}$ be a finite regular cell complex (cf. [4, Section 6.2]). We use the convention that $\emptyset \in \mathcal{X}$. Here the adjective "regular" means that the closure $\bar{\sigma} \subset X$ of an $i$-cell $\emptyset \neq \sigma \in \mathcal{X}$ is always homeomorphic to the closed ball $\left\{x \in \mathbb{R}^{i} \mid\|x\| \leq 1\right\}$. If we regard a finite simplicial complex as a cell complex, then it is regular.

We say $\mathcal{X}$ satisfies the intersection property, if for each $\sigma, \tau \in \mathcal{X}$, there is a cell $v \in \mathcal{X}$ such that $\bar{v}=\bar{\sigma} \cap \bar{\tau}$ (here, $v$ can be $\emptyset$ ). Simplicial complexes and the boundary complexes of convex polytopes satisfy this property.

Clearly, the underlying space $X$ of $\mathcal{X}$ has a finite simplicial decomposition. We say $\mathcal{X}$ is Cohen-Macaulay over a field $\mathbb{k}$ if the Stanley-Reisner ring $\mathbb{k}[\Delta]$ is CohenMacaulay for some (equivalently, all) finite simplicial decomposition $\Delta$ of $X$. The Cohen-Macaulay property of $\mathbb{k}[\Delta]$ may depend on char $(\mathbb{k})$. However, since we fix the base field $\mathbb{k}$ throughout this paper, we often omit the phrase "over $\mathbb{k}$ ". We can define the Buchsbaum property of $\mathcal{X}$ in the same way.

Let $V:=\{\sigma \in \mathcal{X} \mid \operatorname{dim} \sigma=0\}$ be the set of vertices of $\mathcal{X}$. For a subset $W \subset V$, set

$$
\left.\mathcal{X}\right|_{W}:=\{\tau \in \mathcal{X} \mid \sigma \subset \bar{\tau}, \sigma \in V \Longrightarrow \sigma \in W\} .
$$

This is a subcomplex of $\mathcal{X}$. We simply denote $\left.\mathcal{X}\right|_{V \backslash W}$ by $\left.\mathcal{X}\right|_{-W}$.

The following notion was introduced by Baclawski ([2]) in the case of simplicial complexes and extended to regular cell complexes with the intersection property by Fløystad ([8]).

Received by the editors January 26, 2010 and, in revised form, August 7, 2010.

2010 Mathematics Subject Classification. Primary 13F55, 13C14; Secondary 55U10.

Key words and phrases. Simplicial complex, regular cell complex, higher Cohen-Macaulay, doubly Cohen-Macaulay, squarefree module, toric face ring, simplicial poset.

The author was partially supported by Grant-in-Aid for Scientific Research (c) (no. 19540028).

(C)2011 American Mathematical Society Reverts to public domain 28 years from publication 3057 
Definition 1.1 (cf. 2, 8]). For a positive integer $l$, we say $\mathcal{X}$ is $l$-Cohen-Macaulay if $\left.\mathcal{X}\right|_{-W}$ is Cohen-Macaulay and $\operatorname{dim} \mathcal{X}=\left.\operatorname{dim} \mathcal{X}\right|_{-W}$ for all $W \subset V$ with $\# W<l$. (Hence $\mathcal{X}$ is 1-Cohen-Macaulay if and only if it is Cohen-Macaulay.)

Recently, Fløystad gave the following striking results.

Theorem 1.2 (Fløystad [8]). Let $\mathcal{X}$ be a finite regular cell complex with the intersection property.

(1) 2-Cohen-Macaulay property of $\mathcal{X}$ is a topological property of the underlying space.

(2) If $\mathcal{X}$ is l-Cohen-Macaulay, then the codimension 1 skeleton $\mathcal{X}^{\prime}:=\{\sigma \mid$ $\operatorname{dim} \sigma \leq \operatorname{dim} \mathcal{X}-1\}$ is $(l+1)$-Cohen-Macaulay.

Motivated by the above result, we study the higher Cohen-Macaulay property (especially, 2-Cohen-Macaulay property) of relatively new notions of combinatorial commutative algebra, such as squarefree modules, toric face rings, and (the face rings of) simplicial posets.

For example, in Theorem 4.4 we show that Theorem 1.2 (2) holds for the corresponding regular cell complex $\Gamma(P)$ of a simplicial poset $P$, while $\Gamma(P)$ does not satisfy the intersection property and Theorem 1.2 (1) is no longer true.

The notion of toric face rings, which generalizes both Stanley-Reisner rings and affine semigroup rings, is studied in (for example) [3, 5, 9. A toric face ring is supported by a finite regular cell complex satisfying the intersection property. In Theorem 3.3. we show that, under the assumption that a toric face ring $R$ is "conewise normal", the supporting cell complex $\mathcal{X}$ of $R$ is 2-Cohen-Macaulay if and only if $\mathcal{X}$ (equivalently $R$ ) is Cohen-Macaulay and the canonical module $\omega_{R}$ of $R$ is generated by its degree 0 part. The corresponding statement does not hold for simplicial posets (at least, certain modification is required).

\section{Higher Cohen-Macaulay property of squarefree modules}

Let $S=\mathbb{k}\left[x_{1}, \ldots, x_{n}\right]$ be a polynomial ring, and regard it as a $\mathbb{Z}^{n}$-graded ring. For $\mathbf{a}=\left(a_{1}, \ldots, a_{n}\right) \in \mathbb{N}^{n}$, set $\operatorname{supp}(\mathbf{a}):=\left\{i \mid a_{i} \neq 0\right\} \subset[n]:=\{1, \ldots, n\}$ and $x^{\mathbf{a}}:=\prod x_{i}^{a_{i}} \in S$. Let gr $S$ be the category of $\mathbb{Z}^{n}$-graded finitely generated $S$ modules and degree preserving $S$-homomorphisms. For $M \in \operatorname{gr} S$ and $\mathbf{a} \in \mathbb{Z}^{n}, M_{\mathbf{a}}$ denotes the degree a component of $M$, and $M(\mathbf{a})$ denotes the shifted module of $M$ with $M(\mathbf{a})_{\mathbf{b}}=M_{\mathbf{a}+\mathbf{b}}$. For $M, N \in \operatorname{gr} S, \operatorname{Hom}_{S}(M, N)$ is a $\mathbb{Z}^{n}$-graded $S$-module with $\left[\operatorname{Hom}_{S}(M, N)\right]_{\mathbf{a}}=\operatorname{Hom}_{\mathrm{gr} S}(M, N(\mathbf{a}))$. Hence $\operatorname{Ext}_{S}^{i}(M, N)$ has a similar $\mathbb{Z}^{n_{-}}$ grading.

Definition 2.1 (15). A $\mathbb{Z}^{n}$-graded $S$-module $M$ is called squarefree if it is finitely generated, $\mathbb{N}^{n}$-graded (i.e., $M=\bigoplus_{\mathbf{a} \in \mathbb{N}^{n}} M_{\mathbf{a}}$ ), and the multiplication map $M_{\mathbf{a}} \ni$ $y \longmapsto x^{\mathbf{b}} y \in M_{\mathbf{a}+\mathbf{b}}$ is bijective for all $\mathbf{a}, \mathbf{b} \in \mathbb{N}^{n}$ with $\operatorname{supp}(\mathbf{a}) \supset \operatorname{supp}(\mathbf{b})$.

Let $\mathrm{Sq} S$ be the full subcategory of gr $S$ consisting of squarefree $S$-modules.

For a simplicial complex $\Delta$ with the vertex set $[n]$, the Stanley-Reisner ring $\mathbb{k}[\Delta]=S / I_{\Delta}$ is a squarefree $S$-module. As shown in [8, 15, 16, 17, the notion of squarefree modules is useful in the study of the Stanley-Reisner rings. We have that $\mathrm{Sq} S$ is an abelian category with enough projectives and injectives and an indecomposable injective is of the form $\mathbb{k}[F]:=S /\left(x_{i} \mid i \notin F\right)$ for $F \subset[n]$.

We say $\mathbf{a} \in \mathbb{N}^{n}$ is squarefree if $a_{i}=0,1$ for all $i$. We sometimes identify a squarefree vector $\mathbf{a} \in \mathbb{N}^{n}$ with its support $\operatorname{supp}(\mathbf{a}) \subset[n]$. For example, in this 
situation, $M_{\operatorname{supp}(\mathbf{a})}$ denotes the homogeneous component $M_{\mathbf{a}}$ of $M \in \operatorname{gr} S$. If $M \in$ Sq $S$, the essential information of $M$ appears in its squarefree part $\bigoplus_{F \subset[n]} M_{F}$.

For $W \subset[n]$ and $M \in \mathrm{Sq} S$, we can regard the $\mathbb{k}$-vector space

$$
\left.M\right|_{W}:=\bigoplus_{\substack{\mathbf{a} \in \mathbb{N}^{n} \\ \operatorname{supp}(\mathbf{a}) \subset W}} M_{\mathbf{a}}
$$

as a squarefree module over the polynomial ring $\left.S\right|_{W}:=\mathbb{k}\left[x_{i} \mid i \in W\right]$. This construction is a special case of [10, Definition 3.26]. We simply denote $\left.M\right|_{[n] \backslash W}$ by $\left.M\right|_{-W}$.

Let $\mathbb{k}[\Delta]$ be the Stanley-Reisner ring of a simplicial complex $\Delta$ with the vertex set $[n]$. Then, for $W \subset[n]$, we have $\left.\mathbb{k}[\Delta]\right|_{W} \cong \mathbb{k}\left[\left.\Delta\right|_{W}\right]$ as modules over $\left.S\right|_{W}$, where $\left.\Delta\right|_{W}:=\{F \in \Delta \mid F \subset W\}$ is a simplicial complex with the vertex set $W$.

Definition 2.2. Let $l$ be a positive integer. We say $M \in \operatorname{Sq} S$ is $l$-Cohen-Macaulay, if for each $W \subset[n]$ with $\# W<l,\left.M\right|_{-W}$ is either the 0 module or a CohenMacaulay module with $\left.\operatorname{dim} M\right|_{-W}=\operatorname{dim} M$.

Remark 2.3. (1) For $F \subset[n]$, set $\omega_{F}:=\mathbb{k}[F](-F)$. Then $\omega_{F}$ is a squarefree module such that $\left(\omega_{F}\right)_{F^{\prime}} \neq 0$ implies $F^{\prime}=F$ (hence if $F \cap W \neq \emptyset$, then $\left.\omega_{F}\right|_{-W}=0$ ). We see that $\omega_{F}$ is $l$-Cohen-Macaulay for all $l$.

(2) If $M \in \operatorname{Sq} S$ satisfies $M_{0} \neq 0$, then $\left.M\right|_{-W} \neq 0$ for all $W \subset[n]$. Hence the Stanley-Reisner ring $\mathbb{k}[\Delta]$ is $l$-Cohen-Macaulay in the sense of Definition 2.2 if and only if $\Delta$ is $l$-Cohen-Macaulay in the usual sense (i.e., Definition 1.1).

Let $M \in \operatorname{gr} S$. For $i \in \mathbb{N}$ and $\mathbf{a} \in \mathbb{Z}^{n}$, set $\beta_{i, \mathbf{a}}^{S}(M):=\operatorname{dim}_{\mathbb{k}}\left[\operatorname{Tor}_{i}^{S}(\mathbb{k}, M)\right]_{\mathbf{a}}$. Then the $\mathbb{Z}^{n}$-graded minimal free resolution of $M$ is of the form

$$
\cdots \longrightarrow \bigoplus_{\mathbf{a} \in \mathbb{Z}^{n}} S(-\mathbf{a})^{\beta_{1, \mathbf{a}}^{S}(M)} \longrightarrow \bigoplus_{\mathbf{a} \in \mathbb{Z}^{n}} S(-\mathbf{a})^{\beta_{0, \mathbf{a}}^{S}(M)} \longrightarrow M \longrightarrow 0 .
$$

Lemma 2.4. For $F \subset W \subset[n]$ and a squarefree module $M$, we have

$$
\beta_{i, F}^{\left.S\right|_{W}}\left(\left.M\right|_{W}\right)=\beta_{i, F}^{S}(M) .
$$

Proof. By [10, Proposition 3.27].

Let $\mathrm{D}^{b}(\operatorname{Sq} S)$ be the bounded derived category of Sq $S$. Set $\mathbf{1}:=(1,1, \ldots, 1) \in$ $\mathbb{N}^{n}$. Then $\omega_{S}:=S(-\mathbf{1})$ is the $\mathbb{Z}^{n}$-graded canonical module of $S$, and its translation $\omega_{S}[n] \in \mathrm{D}^{b}(\mathrm{Sq} S)$ gives a normalized $\mathbb{Z}^{n}$-graded dualizing complex of $S$. If $M$ is squarefree, then so is $\operatorname{Ext}_{S}^{i}\left(M, \omega_{S}\right)$. Hence $\mathbf{D}(-):=\operatorname{RHom}_{S}\left(-, \omega_{S}[n]\right)$ gives a functor $\mathrm{D}^{b}(\mathrm{Sq} S) \rightarrow \mathrm{D}^{b}(\mathrm{Sq} S)^{\text {op }}$. Note that $H^{-i}(\mathbf{D}(M))$ is isomorphic to $\operatorname{Ext}_{S}^{n-i}\left(M, \omega_{S}\right)$. As shown in [16], $\mathbf{D}(M) \in \mathrm{D}^{b}(\mathrm{Sq} S)$ is quasi-isomorphic to the complex of the form

$$
\cdots \rightarrow \bigoplus_{\substack{F \subset[n] \\ \# F=i}}\left(M_{F}\right)^{*} \otimes_{\mathbb{k}} \mathbb{k}[F] \rightarrow \bigoplus_{\substack{F \subset[n] \\ \# F=i-1}}\left(M_{F}\right)^{*} \otimes_{\mathbb{k}} \mathbb{k}[F] \rightarrow \cdots \rightarrow\left(M_{0}\right)^{*} \otimes_{\mathbb{k}} \mathbb{k} \rightarrow 0,
$$

where $\left(M_{F}\right)^{*}$ denotes the dual $\mathbb{k}$-vector space of $M_{F}$.

If $\mathbb{k}[\Delta]$ is Buchsbaum (especially Cohen-Macaulay) and $d=\operatorname{dim} \mathbb{k}[\Delta]$, then $\omega_{\mathbb{k}[\Delta]}:=\operatorname{Ext}_{S}^{n-d}\left(\mathbb{k}[\Delta], \omega_{S}\right)$ is called the canonical module of $\mathbb{k}[\Delta]$. This is a squarefree module. In the following sections, we will study a few generalizations of StanleyReisner rings. The canonical modules of these rings are defined in a similar way.

As shown in 10, 12, we can also define the Alexander duality functor $\mathbf{A}: \mathrm{Sq} S \rightarrow$ $(\mathrm{Sq} S)^{\mathrm{op}}$ as follows: For $M \in \mathrm{Sq} S, \mathbf{A}(M)_{F}$ is the dual $\mathbb{k}$-vector space of $M_{[n] \backslash F}$, 
and the multiplication map $\mathbf{A}(M)_{F} \ni y \longmapsto x_{i} y \in \mathbf{A}(M)_{F \cup\{i\}}$ for $i \notin F$ is the $\mathbb{k}$-dual of $M_{[n] \backslash(F \cup\{i\})} \ni z \longmapsto x_{i} z \in M_{[n] \backslash F}$.

The next result is just a module version of [8, Theorem 2.5]. However, it has an application to (the face ring of) a simplicial poset.

Theorem 2.5 (cf. [8, Theorem 2.5]). Let $M$ be a Cohen-Macaulay squarefree $S$-module with $\operatorname{dim} M=d$. For $l \geq 2$, the following are equivalent:

(i) $M$ is l-Cohen-Macaulay.

(ii) $\beta_{i, F}^{S}(M)=0$ for all $F \subset[n]$ and $i \in \mathbb{N}$ such that $i>n-d-l+1$ and $\# F<i+d$.

(iii) $\beta_{i, F}^{S}\left(\operatorname{Ext}_{S}^{n-d}\left(M, \omega_{S}\right)\right)=0$ for all $F \subset[n]$ and $i \in \mathbb{N}$ such that $i<l-1$ and $\# F>i$.

(iv) $\mathbf{A}\left(\operatorname{Ext}_{S}^{n-d}\left(M, \omega_{S}\right)\right)$ satisfies Serre's condition $\left(S_{l-1}\right)$. (For the definition of Serre's condition, see [7.)

(v) $\mathbf{A}\left(\operatorname{Ext}_{S}^{n-d}\left(M, \omega_{S}\right)\right)$ is the $(l-1)$-st syzygy module of some $S$-module.

Proof. (i) $\Leftrightarrow$ (ii): Since $\left.M\right|_{W}$ is a squarefree module over $\left.S\right|_{W}, \beta_{i, \mathbf{a}}^{\left.S\right|_{W}}\left(\left.M\right|_{W}\right) \neq 0$ implies that $\mathbf{a}$ is a squarefree vector. Hence, $\left.M\right|_{W}$ is either the 0 module or a CohenMacaulay module of dimension $d$ if and only if $\beta_{i, F}^{\left.S\right|_{W}}\left(\left.M\right|_{W}\right)=0$ for all $F \subset W$ and $i \in \mathbb{N}$ with $i>\# W-d$. Hence the assertion follows from Lemma 2.4 and easy computation.

(ii) $\Leftrightarrow$ (iii): Let $P_{\bullet}$ be a $\mathbb{Z}^{n}$-graded minimal $S$-free resolution of $M$. Since $\omega_{S} \cong S(-\mathbf{1})$ and $M$ is Cohen-Macaulay, $\operatorname{Hom}_{S}\left(P_{\bullet}, \omega_{S}\right)$ is a $\mathbb{Z}^{n}$-graded minimal free resolution of $\operatorname{Ext}_{S}^{n-d}\left(M, \omega_{S}\right)$ after suitable translation. Hence we have

$$
\beta_{i, F}^{S}(M)=\beta_{n-d-i,[n] \backslash F}^{S}\left(\operatorname{Ext}_{S}^{n-d}\left(M, \omega_{S}\right)\right) .
$$

Now the equivalence is clear.

When $M=\mathbb{k}[\Delta]$, the equivalence (iii) $\Leftrightarrow$ (iv) has been proved in [15, Corollary 3.7]. If an $S$-module $N$ satisfies $\left(S_{i}\right)$ for $i \geq 1$, then $\operatorname{Ass}(N)=\operatorname{Ass}(S)=\{(0)\}$ and $\operatorname{dim} N=n$. Hence the proof of [15, Corollary 3.7] also works here (but use [16, Corollary 3.6], which is a squarefree module version of [15, Theorem 3.4]).

The equivalence (iv) $\Leftrightarrow(\mathrm{v})$ is nothing other than (a special case of) [7, Theorem 3.8], which is a classical result essentially due to Auslander and Bridger.

The following is a special case of the equivalence (i) $\Leftrightarrow$ (iii) of Theorem 2.5 However, this fact will be mentioned repeatedly in the following sections.

Corollary 2.6. Let $M$ be a Cohen-Macaulay squarefree $S$-module with $\operatorname{dim} M=d$. Then $M$ is 2-Cohen-Macaulay if and only if $\operatorname{Ext}_{S}^{n-d}\left(M, \omega_{S}\right)$ is generated by its degree 0 part.

For $M \in \operatorname{Sq} S$ and $i \in \mathbb{Z}$ with $0 \leq i<d:=\operatorname{dim} M$, the submodule

$$
M^{>i}:=\bigoplus_{\substack{\mathbf{a} \in \mathbb{N}^{n} \\ \# \operatorname{supp}(\mathbf{a})>i}} M_{\mathbf{a}}
$$

of $M$ is a squarefree module again. Set $M^{\langle i\rangle}:=M / M^{>i}$. Clearly, $M^{\langle i\rangle} \in \mathrm{Sq} S$ and $\operatorname{dim} M^{\langle i\rangle} \leq i\left(M^{\langle i\rangle}\right.$ can be the 0 module). For a simplicial complex $\Delta$ with the vertex set $[n]$, let $\Delta^{(i)}=\{F \in \Delta \mid \# F \leq i+1\}$ be its $i$-skeleton. Then, as an $S$-module, we have $\mathbb{k}\left[\Delta^{(i)}\right] \cong \mathbb{k}[\Delta]^{\langle i+1\rangle}$ (note that $\operatorname{dim} \mathbb{k}[\Delta]=\operatorname{dim} \Delta+1$ ). 
Theorem 2.7 (cf. [8, Corollary 2.7]). Let $M$ be a squarefree $S$-module of dimension $d$, and let $i$ be an integer with $0 \leq i<d$. If $M$ is l-Cohen-Macaulay, then $M^{\langle i\rangle}$ is $(l+d-i)$-Cohen-Macaulay (unless $\left.M^{\langle i\rangle}=0\right)$.

Proof. Since $M$ is Cohen-Macaulay, only non-vanishing cohomology of the complex $\mathbf{D}(M)$ is $H^{-d}(\mathbf{D}(M))$, which is isomorphic to $\operatorname{Ext}_{S}^{n-d}\left(M, \omega_{S}\right)$. If we use (2.1) for a description of the complex $D^{\bullet}:=\mathbf{D}(M)$, then $\mathbf{D}\left(M^{\langle i\rangle}\right)$ is the brutal truncation $\cdots \rightarrow 0 \rightarrow D^{-i} \rightarrow D^{-i+1} \rightarrow D^{-i+2} \rightarrow \cdots$ of $D^{\bullet}$. Hence $H^{j}\left(\mathbf{D}\left(M^{\langle i\rangle}\right)\right)=0$ for all $j \neq-i$, that is, $M^{\langle i\rangle}$ is Cohen-Macaulay. Since $H^{-i}\left(\mathbf{D}\left(M^{\langle i\rangle}\right) \cong \operatorname{Ext}_{S}^{n-i}\left(M^{\langle i\rangle}, \omega_{S}\right)\right.$, we have the following exact sequences:

$$
0 \rightarrow \operatorname{Ext}_{S}^{n-d}\left(M, \omega_{S}\right) \rightarrow D^{-d} \rightarrow D^{-d+1} \rightarrow \cdots \rightarrow D^{-i+1} \rightarrow \operatorname{Ext}_{S}^{n-i}\left(M^{\langle i\rangle}, \omega_{S}\right) \rightarrow 0
$$

and

$$
0 \rightarrow \mathbf{A}\left(\operatorname{Ext}_{S}^{n-i}\left(M^{\langle i\rangle}, \omega_{S}\right)\right) \rightarrow \mathbf{A}\left(D^{-i+1}\right) \rightarrow \cdots \rightarrow \mathbf{A}\left(D^{-d}\right) \rightarrow \mathbf{A}\left(\operatorname{Ext}_{S}^{n-d}\left(M \omega_{S}\right)\right) \rightarrow 0 .
$$

Since $\mathbf{A}(\mathbb{k}[F])$ is isomorphic to $S(-([n] \backslash F)), \mathbf{A}\left(D^{j}\right)$ is a free $S$-module for all $j$. Hence $\mathbf{A}\left(\operatorname{Ext}_{S}^{n-i}\left(M^{\langle i\rangle}, \omega_{S}\right)\right)$ is the $(d-i)$ th syzygy of $\mathbf{A}\left(\operatorname{Ext}_{S}^{n-d}\left(M, \omega_{S}\right)\right)$. Hence the assertion follows from the equivalence between (i) and (v) of Theorem 2.5.

\section{Toric face Rings and 2-Cohen-Macaulay Cell Complexes}

While we discuss toric face rings in this section, we only give a "casual" definition/construction of this ring and some related notions. See [11] for precise information. The original construction found in [5] is equivalent to that of [1], but does not mention the regular cell complex supporting a toric face ring.

A toric face ring is constructed from a monoidal complex $\mathcal{M}$ supported by a finite regular cell complex $\mathcal{X}$ with the intersection property. Here $\mathcal{M}$ is a collection $\left\{\mathbb{M}_{\sigma}\right\}_{\sigma \in \mathcal{X}}$ of affine semigroups $\mathbb{M}_{\sigma} \subset \mathbb{Z}^{\operatorname{dim} \sigma+1}$ (i.e., $\mathbb{M}_{\sigma}$ is a finitely generated additive submonoid of $\left.\mathbb{Z}^{\operatorname{dim} \sigma+1}\right)$ with $\mathbb{Z} \mathbb{M}_{\sigma}=\mathbb{Z}^{\operatorname{dim} \sigma+1}$ and $\mathbb{M}_{\sigma} \cap\left(-\mathbb{M}_{\sigma}\right)=\{0\}$. Of course, we require several conditions on $\mathbb{M}_{\sigma}$ 's (not all finite regular cell complexes with the intersection property can support a monoidal complex). We assume that the boundary complex of the cross section $\overline{\mathcal{P}}_{\sigma}$ of the polyhedral cone $\mathcal{P}_{\sigma}:=\mathbb{R}_{\geq 0} \mathbb{M}_{\sigma} \subset \mathbb{R}^{\operatorname{dim} \sigma+1}$ can be identified with the subcomplex $\{\tau \mid \tau \subset \bar{\sigma}\}$ of $\mathcal{X}$ (note that $\overline{\mathcal{P}}_{\sigma}$ is a convex polytope of dimension $\operatorname{dim} \sigma$ ). The face $\mathcal{C}_{\tau}$ of $\mathcal{P}_{\sigma}$ corresponding to $\tau \subset \bar{\sigma}$ is isomorphic to $\mathcal{P}_{\tau}$ as a polyhedral cone. Moreover, the monoid $\mathbb{M}_{\tau}$ is isomorphic to $\mathcal{C}_{\tau} \cap \mathbb{M}_{\sigma}$.

Let $|\mathcal{M}|$ be the set given by glueing all $\mathbb{M}_{\sigma}$ 's along with $\mathcal{X}$. We can regard $\mathbb{M}_{\sigma} \subset|\mathcal{M}|$ for all $\sigma \in \mathcal{X}$. For $a, b \in|\mathcal{M}|$, we cannot define their sum in general; that is, $|\mathcal{M}|$ is no longer a monoid. However, if there is some $\sigma \in \mathcal{X}$ with $a, b \in \mathbb{M}_{\sigma}$, then we have their sum $a+b \in \mathbb{M}_{\sigma} \subset|\mathcal{M}|$.

Then the toric face ring $R:=\mathbb{k}[\mathcal{M}]$ of $\mathcal{M}$ over $\mathbb{k}$ is the vector space $\bigoplus_{a \in|\mathcal{M}|} \mathbb{k} t^{a}$ with the $\mathbb{k}$-linear multiplication defined by

$$
t^{a} \cdot t^{b}= \begin{cases}t^{a+b} & \text { if } a, b \in \mathbb{M}_{\sigma} \text { for some } \sigma \in \mathcal{X}, \\ 0 & \text { otherwise. }\end{cases}
$$

Note that $\operatorname{dim} R=\operatorname{dim} \mathcal{X}+1$.

For each $\sigma \in \mathcal{X}, \mathfrak{p}_{\sigma}:=\left(t^{a} \mid a \notin \mathbb{M}_{\sigma}\right)$ is a prime ideal of $R$ with $R / \mathfrak{p}_{\sigma} \cong \mathbb{k}\left[\mathbb{M}_{\sigma}\right]$, where $\mathbb{k}\left[\mathbb{M}_{\sigma}\right]$ is the semigroup ring of $\mathbb{M}_{\sigma}$. Clearly, $\mathbb{k}\left[\mathbb{M}_{\sigma}\right]$ can be seen as a subring of $R$. In $R, \mathbb{k}\left[\mathbb{M}_{\sigma}\right]$ for $\sigma \in \mathcal{X}$ are "glued" along with $\mathcal{X}$. We say $\mathcal{M}$ is cone-wise normal if $\mathbb{k}\left[\mathbb{M}_{\sigma}\right]$ is normal (equivalently, $\mathbb{Z}^{\operatorname{dim} \sigma+1} \cap \mathbb{R}_{\geq 0} \mathbb{M}_{\sigma}=\mathbb{M}_{\sigma}$ ) for all $\sigma \in \mathcal{X}$. 
Example 3.1. Let $A:=\mathbb{k}\left[x^{\mathbf{a}} \mid \mathbf{a} \in \mathbb{M}\right] \subset \mathbb{k}\left[x_{1}, \ldots, x_{n}\right]$ be the semigroup ring of an affine semigroup $\mathbb{M} \subset \mathbb{N}^{n}$. An easy example of a toric face ring is the quotient ring $A / I$ of $A$ by a radical $\mathbb{Z}^{n}$-graded ideal $I$. Let $\mathcal{P}:=\mathbb{R}_{\geq 0} \mathbb{M} \subset \mathbb{R}^{n}$ be the polyhedral cone spanned by $\mathbb{M}$, and let $L$ be its face lattice. For the ideal $I$, there is a subset $\Sigma$ of $L$ such that

$$
A / I=\bigoplus_{\substack{\mathbf{a} \in \mathcal{C} \cap \mathbb{N}^{n} \\ \mathcal{C} \in \Sigma}} \mathbb{k} x^{\mathbf{a}}
$$

(Note that $\mathcal{C} \in \Sigma$ is a face of $\mathcal{P}$, and it is also a cone in $\mathbb{R}^{n}$.) Clearly, $\{\mathcal{C}\}_{\mathcal{C} \in \Sigma}$ forms a polyhedral fan; that is, $\mathcal{C}^{\prime} \subset \mathcal{C} \in \Sigma$ and $\mathcal{C}^{\prime} \in L$ imply that $\mathcal{C}^{\prime} \in \Sigma$. The monoidal complex giving $A / I$ is $\mathcal{M}:=\{\mathbb{M} \cap \mathcal{C} \mid \mathcal{C} \in \Sigma\}$. Let $H \subset \mathbb{R}^{n}$ be a hyperplane intersecting $\mathcal{P}$ transversely. The cell complex supporting $\mathcal{M}$ is given by $\{\operatorname{rel}$-int $(H \cap \mathcal{C}) \mid \mathcal{C} \in \Sigma\}$. If $A$ is normal, then $A / I$ is cone-wise normal.

If $A$ is the polynomial ring $\mathbb{k}\left[x_{1}, \ldots, x_{n}\right]$, then $A / I$ can be attained as the StanleyReisner ring $\mathbb{k}[\Delta]$ of a simplicial complex $\Delta$. In this case, the supporting cell complex is nothing other than $\Delta$.

Clearly, $A / I$ of the above example has a $\mathbb{Z}^{n}$-grading inherited from that of $A$. However, a toric face ring does not admit a nice multi-grading in its most general setting, while the decomposition $R=\bigoplus_{a \in|\mathcal{M}|} \mathbb{k} t^{a}$ plays a similar role to the graded structure.

Known results. Let $\mathcal{M}$ be a cone-wise normal monoidal complex supported by a cell complex $\mathcal{X}$. For $R:=\mathbb{k}[\mathcal{M}]$, we have the following. See [11] for details.

(1) $R$ is Cohen-Macaulay (resp. Buchsbaum) if and only if $\mathcal{X}$ is Cohen-Macaulay (resp. Buchsbaum) in the sense of $\S 1$.

(2) We can naturally define squarefree modules over $R$. For example, $R$ itself is squarefree. If $R$ is Buchsbaum, then the canonical module $\omega_{R}$ of $R$ is also Buchsbaum.

A squarefree $R$-module $M$ has the decomposition $M=\bigoplus_{a \in|\mathcal{M}|} M_{a}$ as a $\mathbb{k}$-vector space. Note that $|\mathcal{M}|$ has the 0 element. In the sequel, the component $M_{0}$ plays a role.

(3) A squarefree $R$-module $M$ gives the constructible sheaf $M^{+}$on the un-

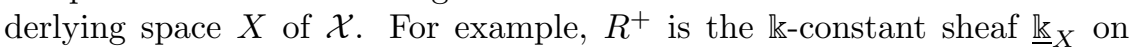
$X$.

Let $\mathcal{M}$ be a cone-wise normal monoidal complex supported by a regular cell complex $\mathcal{X}$ with $d=\operatorname{dim} \mathcal{X}$. Then the underlying space $X$ of $\mathcal{X}$ admits the Verdier dualizing complex $\mathcal{D}_{X}^{\bullet}$ with the coefficients in $\mathbb{k}$. By [11, Theorem 6.4], $R:=\mathbb{k}[\mathcal{M}]$ is Buchsbaum if and only if $\mathcal{H}^{i}\left(\mathcal{D}_{X}^{\bullet}\right)=0$ for all $i \neq-d$. In this case, set

$$
\mathcal{W}_{X}:=\mathcal{H}^{-d}\left(\mathcal{D}_{X}^{\bullet}\right) \text {. }
$$

If $X$ is a manifold, then $R$ is Buchsbaum and $\mathcal{W}_{X}$ is the orientation sheaf of $X$ with the coefficients in $\mathbb{k}$. Moreover, $\left(\omega_{R}\right)^{+} \cong \mathcal{W}_{X}$.

Lemma 3.2. Let $R$ be a cone-wise normal toric face ring, and $M$ a squarefree module over $R$ with $\operatorname{depth}_{R} M \geq 2$. Then $M$ is generated by its degree 0 part $M_{0}$ if and only if the sheaf $M^{+}$is generated by global sections.

Proof. For each $\emptyset \neq \sigma \in \mathcal{X}$, take an element $a(\sigma) \in \mathbb{M}_{\sigma} \subset|\mathcal{M}|$ contained in the interior of the cone $\mathbb{R}_{\geq 0} \mathbb{M}_{\sigma}$ in $\mathbb{R}^{\operatorname{dim} \sigma+1}$. The stalk $\left(M^{+}\right)_{p}$ at any point $p \in \sigma$ 
is isomorphic to $M_{a(\sigma)}$. Since $\operatorname{depth}_{R} M \geq 2$, we have $\Gamma\left(X, M^{+}\right) \cong M_{0}$ by [11, Theorem 6.2 (a)], and the natural map $\Gamma\left(X, M^{+}\right) \rightarrow\left(M^{+}\right)_{p}$ corresponds to the map $\varphi_{\sigma}: M_{0} \ni x \longmapsto t^{a(\sigma)} x \in M_{a(\sigma)}$. Hence $M^{+}$is generated by global sections if and only if the map $\varphi_{\sigma}$ is surjective for all $\sigma \neq \emptyset$. Since $M$ is squarefree, the latter condition states that $M$ is generated by $M_{0}$ as an $R$-module.

Theorem 3.3. Le $\mathcal{M}$ be a cone-wise normal monoidal complex supported by a cell complex $\mathcal{X}$ with the underlying space $X$. For the toric face ring $R:=\mathbb{k}[\mathcal{M}]$, the following are equivalent:

(i) $\mathcal{X}$ is 2-Cohen-Macaulay.

(ii) $R$ is Cohen-Macaulay, and the canonical module $\omega_{R}$ is generated by its degree 0 part.

If $\operatorname{dim} R \geq 2$, the above conditions are also equivalent to the following:

(iii) $\mathcal{X}$ is Cohen-Macaulay and the sheaf $\mathcal{W}_{X}$ is generated by global sections.

Proof. A toric face ring of dimension 1 (i.e., the case when $\operatorname{dim} \mathcal{X}=0$ ) is always a Stanley-Reisner ring. Moreover, in this case, $\mathcal{X}$ is 2-Cohen-Macaulay unless $\mathcal{X}$ consists of a single point. Hence the assertion is easy if $\operatorname{dim} R=1$.

So it suffices to show the equivalence of (i), (ii), and (iii) under the assumption that $\operatorname{dim} R \geq 2$. Now $\operatorname{depth}_{R} \omega_{R} \geq 2$. Since $\mathcal{W}_{X} \cong\left(\omega_{R}\right)^{+}$, the equivalence (ii) $\Leftrightarrow$ (iii) follows from Lemma 3.2 It remains to prove the equivalence (i) $\Leftrightarrow$ (iii). Let $\Delta$ be the barycentric subdivision of $\mathcal{X}$. Since $\mathcal{X}$ satisfies the intersection property, $\mathcal{X}$ is 2-Cohen-Macaulay if and only if $\Delta$ is 2-Cohen-Macaulay by [8, Theorem 2.8]. On the other hand, as shown in 2 (and as a special case of Corollary 2.6), $\Delta$ is 2-CohenMacaulay if and only if $\Delta$ is Cohen-Macaulay and the canonical module $\omega_{\mathbb{k}}[\Delta]$ of the Stanley-Reisner ring $\mathbb{k}[\Delta]$ is generated by its degree 0 part. Since $\operatorname{depth} \omega_{\mathbb{k}[}[\Delta] \geq 2$ and $\left(\omega_{\mathbb{k}[\Delta]}\right)^{+} \cong \mathcal{W}_{X}$, the desired equivalence follows from Lemma 3.2

Remark 3.4. Let $\mathcal{X}$ be a finite regular cell complex with the intersection property, and set $V:=\{\sigma \in \mathcal{X} \mid \operatorname{dim} \sigma=0\}$ and $d:=\operatorname{dim} \mathcal{X}$. Fløystad ([8]) constructed the $i$ th enriched cohomology $\mathbf{H}^{i}(\mathcal{X} ; \mathbb{k})$ (or just $\mathbf{H}^{i}(\mathcal{X})$, since we have fixed the base field $\mathbb{k})$ of $\mathcal{X}$, which is a squarefree module over the polynomial ring $S:=\mathbb{k}\left[x_{\sigma} \mid \sigma \in V\right]$. If $\Delta$ is a simplicial complex, we have $\mathbf{A}\left(\mathbf{H}^{i}(\Delta)\right) \cong \operatorname{Ext}_{S}^{\# V-i-1}\left(\mathbb{k}[\Delta], \omega_{S}\right)$ for all $i$. Even for general $\mathcal{X}$, it is Cohen-Macaulay if and only if $\mathbf{H}^{i}(\mathcal{X})=0$ for all $i \neq d$.

From now on, we assume that $\mathcal{X}$ is Cohen-Macaulay. [8, Theorem 2.4] states that $\mathcal{X}$ is $l$-Cohen-Macaulay if and only if $\mathbf{H}^{d}(\mathcal{X})$ is the $(l-1)$-st syzygy module of some $S$-module. Hence $\mathcal{X}$ is 2-Cohen-Macaulay if and only if $\mathbf{A}\left(\mathbf{H}^{d}(\mathcal{X})\right)$ is generated by its degree 0 part. Clearly, this is analogous to Theorem 3.3. However, the relation between $\mathbf{A}\left(\mathbf{H}^{d}(\mathcal{X})\right)$ and $\omega_{R}$ is not direct unless $\mathcal{X}$ is a simplicial complex.

\section{Simplicial POSET}

A finite partially ordered set (poset, for short) $P$ is called simplicial if it admits the smallest element $\hat{0}$, and the interval $[\hat{0}, x]:=\{y \in P \mid y \leq x\}$ is isomorphic to a boolean algebra for all $x \in P$. For the simplicity, we denote $\operatorname{rank}(x)$ of $x \in P$ by $\rho(x)$. If $P$ is simplicial and $\rho(x)=m$, then $[\hat{0}, x]$ is isomorphic to the boolean algebra $2^{\{1, \ldots, m\}}$.

Let $\Delta$ be a finite simplicial complex (with $\emptyset \in \Delta$ ). Its face poset (i.e., the set of the faces of $\Delta$ with the order given by inclusion) is a simplicial poset. Any simplicial poset $P$ is the face (cell) poset of a regular cell complex, which we denote 
by $\Gamma(P)$. Unless $\Gamma(P)$ is a simplicial complex, it does not satisfy the intersection property. For example, if two $d$-simplices are glued along their boundaries, then it is not a simplicial complex, but gives a simplicial poset.

From now on, let $P$ be a simplicial poset. For $x, y \in P$, set

$$
[x \vee y]:=\text { the set of minimal elements of }\{z \in P \mid z \geq x, y\} \text {. }
$$

More generally, for $x_{1}, \ldots, x_{m} \in P,\left[x_{1} \vee \cdots \vee x_{m}\right]$ denotes the set of minimal elements of the common upper bounds of $x_{1}, \ldots, x_{m}$.

Set $V:=\{y \in P \mid \rho(y)=1\}=\left\{y_{1}, \ldots, y_{n}\right\}$. We sometimes identify $V$ with $[n]$ in the natural way. For $U \subset V$, we simply denote $\left[\bigvee_{i \in U} y_{i}\right]$ by $[U]$. Then we have $P=\bigsqcup_{U \subset V}[U]$. For $W \subset V$, set

$$
\left.P\right|_{W}:=\coprod_{U \subset W}[U] .
$$

Clearly, $\left.P\right|_{W}$ is simplicial again. We simply denote $\left.P\right|_{V \backslash W}$ by $\left.P\right|_{-W}$.

Stanley [13] defined the face ring $A_{P}$ of a simplicial poset $P$. For the definition, we remark that if $[x \vee y] \neq \emptyset$, then $\{z \in P \mid z \leq x, y\}$ has the largest element, which is denoted by $x \wedge y$. Let $S:=\mathbb{k}\left[t_{x} \mid x \in P\right]$ be the polynomial ring in the variables $t_{x}$. Consider the ideal

$$
I_{P}:=\left(t_{x} t_{y}-t_{x \wedge y} \sum_{z \in[x \vee y]} t_{z} \mid x, y \in P\right)+\left(t_{\hat{0}}-1\right)
$$

of $S$ (if $[x \vee y]=\emptyset$, we interpret that $t_{x \wedge y} \cdot \sum_{z \in[x \vee y]} t_{z}=0$ ), and set

$$
A_{P}:=S / I_{P}
$$

We have $\operatorname{dim} A_{P}=\operatorname{rank} P=\operatorname{dim} \Gamma(P)+1$.

For a rank 1 element $y_{i} \in V$, we simply denote $t_{y_{i}}$ by $t_{i}$. If $\{x\}=[U]$ for some $U \subset V$ with $\# U \geq 2$, then $t_{x}=\prod_{i \in U} t_{i}$ in $A_{P}$, and $t_{x}$ is a "dummy". Since $I_{P}$ is a homogeneous ideal under the grading given by $\operatorname{deg}\left(t_{x}\right)=\rho(x), A_{P}$ is a graded ring. The algebra $A_{P}$ is generated by degree 1 elements if and only if $\Gamma(P)$ is a simplicial complex. In this case, $A_{P}$ coincides with the Stanley-Reisner ring of $\Gamma(P)$.

We say $P$ is Cohen-Macaulay (resp. Buchsbaum) if the cell complex $\Gamma(P)$ is Cohen-Macaulay (resp. Buchsbaum) in the sense of $\S 1$. Duval [6] showed that $P$ is Cohen-Macaulay if and only if $A_{P}$ is a Cohen-Macaulay ring. The same is true for the Buchsbaum property (cf. [17]).

Proposition 4.1. Some (equivalently, all) finite simplicial decompositions of $\Gamma(P)$ are 2-Cohen-Macaulay if and only if $A:=A_{P}$ is Cohen-Macaulay and the canonical module $\omega_{A}$ is generated by its degree 0 part.

Proof. We can define squarefree modules over $A$ in a natural way, and a squarefree $A$-module $M$ gives the constructible sheaf $M^{+}$on $\Gamma(P)$ (see [17]). The theory of squarefree modules over $A$ is quite parallel to that over a toric face ring. Hence we can prove the assertion by the same method as in Theorem 3.3 .

Note that $V=\{y \in P \mid \rho(y)=1\}$ can be identified with the vertex set of the cell complex $\Gamma(P)$, and $\left.\Gamma(P)\right|_{-W}=\Gamma\left(\left.P\right|_{-W}\right)$ for $W \subset V$. Hence $\Gamma(P)$ is $l$-CohenMacaulay (in the sense of Definition 1.1) if and only if $\left.P\right|_{-W}$ is Cohen-Macaulay and $\operatorname{rank}\left(\left.P\right|_{-W}\right)=\operatorname{rank} P$ for all $W \subset V$ with $\# W<l$.

Recall our convention that $V=\left\{y_{1}, \ldots, y_{n}\right\}$ and $t_{i}:=t_{y_{i}}$. Clearly, $A:=A_{P}$ has a $\mathbb{Z}^{n}$-grading such that $\operatorname{deg} t_{i} \in \mathbb{N}^{n}$ is the $i$ th unit vector. Consider the polynomial 
$\operatorname{ring} T:=\operatorname{Sym}\left(A_{1}\right) \cong \mathbb{k}\left[t_{1}, \ldots, t_{n}\right]$. Then $A$ is a finitely generated $\mathbb{Z}^{n}$-graded $T$ module; moreover, $A$ is a squarefree $T$-module. If $A$ is Buchsbaum, then $\omega_{A}$ is a squarefree $T$-module.

Let $W \subset V$. If we regard the face ring $A_{\left.P\right|_{W}}$ of $\left.P\right|_{W}$ as a module over the polynomial ring $\left.T\right|_{W}=\mathbb{k}\left[t_{i} \mid i \in W\right]$, it coincides with the restriction $\left.\left(A_{P}\right)\right|_{W}$ of $A_{P}$ as a squarefree $T$-module (see $\S 2$ ). As in Remark 2.3, $\Gamma(P)$ is $l$-Cohen-Macaulay if and only if $A_{P}$ is $l$-Cohen-Macaulay as a squarefree $T$-module.

Proposition 4.2. Let the notation be as above. The cell complex $\Gamma(P)$ is 2-CohenMacaulay if and only if $A:=A_{P}$ is Cohen-Macaulay and the canonical module $\omega_{A}$ is generated by its degree 0 part as a T-module.

In particular, if $\Gamma(P)$ is 2-Cohen-Macaulay, then some (equivalently, all) finite simplicial decomposition of $\Gamma(P)$ is 2-Cohen-Macaulay.

Proof. The first assertion follows from Corollary 2.6. The second follows from the first and Proposition 4.1.

Remark 4.3. Even if a finite simplicial decomposition of $\Gamma(P)$ is 2-Cohen-Macaulay, $\Gamma(P)$ itself is not, in general. For example, let $P$ be the simplicial poset given by two $d$-simplices glued along their boundaries. Since the underlying space of $\Gamma(P)$ is a $d$ dimensional sphere, its simplicial decompositions are 2-Cohen-Macaulay. However, $\operatorname{rank}\left(\left.P\right|_{-\{y\}}\right)<\operatorname{rank} P$ for all $y \in V$, and $\Gamma(P)$ is not 2-Cohen-Macaulay.

Consider the induced subposet $P^{\langle i\rangle}:=\{x \in P \mid \rho(x) \leq i\}$ of $P$. This is a simplicial poset again.

Theorem 4.4 (cf. [8, Corollary 2.7]). Let $P$ be a simplicial poset with $\operatorname{rank} P=d$. If $\Gamma(P)$ is l-Cohen-Macaulay, then $\Gamma\left(P^{\langle i\rangle}\right)$ is $(l+d-i)$-Cohen-Macaulay.

Proof. Recall that $A_{P}$ is a squarefree module over $T=\mathbb{k}\left[t_{1}, \ldots, t_{n}\right]$. The face ring $A_{P\langle i\rangle}$ of $P^{\langle i\rangle}$ coincides with the skeleton $\left(A_{P}\right)^{\langle i\rangle}$ of $A_{P}$ as a squarefree $T$-module. Hence the assertion follows from Theorem 2.7

The next statement immediately follows from Theorem 4.4 Let $P$ be a CohenMacaulay simplicial poset with $\operatorname{rank} P=d$. Then the edge graph of $P$ (i.e., the skeleton $\left.P^{\langle 2\rangle}\right)$ is $(d-1)$-connected. However, this also follows from [6, Theorem 4.5].

Remark 4.5 (Buchsbaum* complex). Athanasiadis and Welker (1]) call a finite simplicial complex $\Delta$ Buchsbaum* (over $\mathbb{k}$ ) if $\mathbb{k}[\Delta]$ is Buchsbaum and the canonical module $\omega_{\mathbb{k}[\Delta]}$ is generated by its degree 0 part. (Their original definition is different, but equivalent to the above one by [1, Proposition 2.8]). Since the Buchsbaum* property is topological, we say a finite regular cell complex $\mathcal{X}$ is Buchsbaum* if some (equivalently, all) finite simplicial decomposition of $\mathcal{X}$ is so. By the same argument as the proof of Theorem 3.3. we have the following:

(1) Let $R$ be a cone-wise normal toric face ring. The supporting cell complex of $R$ is Buchsbaum* if and only if $R$ is Buchsbaum and the canonical module $\omega_{R}$ is generated by its degree 0 part.

(2) Let $P$ be a simplicial poset and $A$ its face ring. Then $\Gamma(P)$ is Buchsbaum* if and only if $A$ is Buchsbaum and $\omega_{A}$ is generated by its degree 0 part (as an $A$-module, of course). 


\section{ACKNOWLEDGMENT}

The author is grateful to Ezra Miller for his helpful comments on an earlier version of this paper.

\section{REFERENCES}

[1] C.A. Athanasiadis and V. Welker, Buchsbaum* complexes, preprint (arXiv:0909.1931).

[2] K. Baclawski, Cohen-Macaulay connectivity and geometric lattices, Europ. J. Combinatorics 3 (1982), 293-305. MR687728 (84d:06001)

[3] W. Bruns and J. Gubeladze, Polytopes, rings, and $K$-theory, Springer, 2009. MR 2508056 (2010d:19001)

[4] W. Bruns and J. Herzog, Cohen-Macaulay rings, revised edition, Cambridge University Press, 1998. MR1251956 (95h:13020)

[5] W. Bruns, R. Koch, and T. Römer, Gröbner bases and Betti numbers of monoidal complexes, Michigan Math. J. 57 (2008), 71-91. MR2492442 (2010a:13045)

[6] A.M. Duval, Free resolutions of simplicial posets, J. Algebra 188 (1997), 363-399. MR1432361 (97m:13031)

[7] E. G. Evans and P. Griffith, Syzygies, London Mathematical Society Lecture Note Series, vol. 106, 1985. MR811636 (87b:13001)

[8] G. Fløystad, Cohen-Macaulay cell complexes, in Algebraic and Geometric Combinatorics, C. A. Athanasiadis et al., eds., Contemporary Mathematics, vol. 423, American Mathematical Society, 2007, pp. 205-220. MR2298759 (2008h:13036)

[9] B. Ichim and T. Römer, On toric face rings, J. Pure Appl. Algebra 210 (2007), 249-266. MR2311184(2008a:13032)

[10] E. Miller, The Alexander duality functors and local duality with monomial support, J. Algebra 231 (2000), 180-234. MR.1779598 (2001k:13028)

[11] R. Okazaki and K. Yanagawa, Dualizing complex of a toric face ring, Nagoya Math. J. 196 (2009), 87-116. MR2591092

[12] T. Römer, Generalized Alexander duality and applications, Osaka J. Math. 38 (2001), 469485. MR:1833633 (2002c:13029)

[13] R. Stanley, f-vectors and h-vectors of simplicial posets, J. Pure Appl. Algebra 71 (1991), 319-331. MR1117642 (93b:06009)

[14] R. Stanley, Combinatorics and commutative algebra, 2nd ed., Birkhäuser, 1996. MR.1453579 (98h:05001)

[15] K. Yanagawa, Alexander duality for Stanley-Reisner rings and squarefree $\mathbb{N}^{n}$-graded modules, J. Algebra 225 (2000), 630-645. MR.1741555 (2000m:13036)

[16] K. Yanagawa, Derived category of squarefree modules and local cohomology with monomial ideal support, J. Math. Soc. Japan 56 (2004), 289-308. MR2028674 (2004j:13041)

[17] K. Yanagawa, Dualizing complex of the face ring of a simplicial poset, preprint (arXiv:0910.1498).

Department of Mathematics, Kansai University, Suita 564-8680, Japan

E-mail address: yanagawa@ipcku.kansai-u.ac.jp 